\title{
Ecosystem Intelligence for AI-based Assistant Platforms
}

\author{
Rainer Schmidt \\ Munich University of \\ Applied Sciences \\ rainer.schmidt@hm.edu
}

\author{
Rainer Alt \\ Leipzig University \\ rainer.alt@uni-leipzig.de
}

\author{
Alfred Zimmermann \\ Reutlingen University \\ alfred.zimmermann@reutlingen- \\ university.de
}

\begin{abstract}
Digital assistants like Alexa, Google Assistant or Siri have seen a large adoption over the past years. Using artificial intelligence (AI) technologies, they provide a vocal interface to physical devices as well as to digital services and have spurred an entire new ecosystem. This comprises the big tech companies themselves, but also a strongly growing community of developers that make these functionalities available via digital platforms. At present, only few research is available to understand the structure and the value creation logic of these AI-based assistant platforms and their ecosystem. This research adopts ecosystem intelligence to shed light on their structure and dynamics. It combines existing data collection methods with an automated approach that proves useful in deriving a network-based conceptual model of Amazon's Alexa assistant platform and ecosystem. It shows that skills are a key unit of modularity in this ecosystem, which is linked to other elements such as service, data, and money flows. It also suggests that the topology of the Alexa ecosystem may be described using the criteria reflexivity, symmetry, variance, strength, and centrality of the skill coactivations. Finally, it identifies three ways to create and capture value on AIbased assistant platforms. Surprisingly only a few skills use a transactional business model by selling services and goods but many skills are complementary and provide information, configuration, and control services for other skill provider products and services. These findings provide new insights into the highly relevant ecosystems of AI-based assistant platforms, which might serve enterprises in developing their strategies in these ecosystems. They might also pave the way to a faster, data-driven approach for ecosystem intelligence.
\end{abstract}

\section{Introduction}

AI-based assistants like Amazon's Alexa, Apple's Siri, Google's Assistant, or Microsoft's Cortana provide a natural language interface for choosing, integrating, and accessing a multitude of services and devices. They have evolved to become platforms in their own since services and devices may originate from different vendors [1]. For example, many devices at home are either accessible via AI-based assistant platforms or have become access points themselves, such as many TV sets [1], and extensive ecosystems with service and device vendors [2] surround AI-based assistant platforms. These ecosystems have become highly relevant for many enterprises to interact with their customers and provide access to their services and devices. On the one hand, these opportunities may create valuable sources of operational efficiency as well as a competitive advantage for enterprises in many industries [3], [4]. On the other hand, it is important to understand the logic of these ecosystems since only 15 percent of the ecosystems thrive in the long run [4]. More than 100 failed ecosystems showed that from seven failures, six were caused by strategic blunders [3]. Developing ecosystem initiatives is difficult because these digitally enabled forms of organization are rather new approaches of economic organization in practice [5] and require new strategic thinking [6]. Ecosystem intelligence has been identified as an important theme of research to understand these organizational forms as well as the associated digital platforms [7]. We postulate the need for ecosystem intelligence on AI-based assistant platforms and formulate the following research questions.

1. Who are the actors in the ecosystem of AI-based assistant platforms and how are they related?

2. What are the topological properties of AI-based assistant platforms and their ecosystems?

3. How is value created and co-created in the ecosystems of AI-based assistant platforms?

This research aims to obtain new insights into the structure of ecosystems of AI-based assistant platforms using the example of Amazon's Alexa ecosystem [8]. Among the key research aspects are the participating actors, the relationships among the actors, the overall topology of the ecosystem as well as the main value (co-)creation processes. The paper is structured as follows. First, we show the status of current research in the field of ecosystems and ecosystem intelligence to substantiate the basic elements of AI-based assistant platforms and their ecosystem. The adopted structured ap- 
proach for ecosystem intelligence based on an automated data collection technique is described in the next section. Then, we elaborate on a network-oriented conceptual model of the ecosystem and describe its structure and relations. The network-oriented conceptual model of the ecosystem enables us to apply established methods from network-based research. After identifying the ecosystem's actors and identify the service, data, and money flows in the ecosystem, the topology of the ecosystem and structural properties of the ecosystem are analyzed. In addition, the value creation and co-creation mechanisms in the ecosystem are analyzed. The findings are then discussed before we conclude and provide outlook on further work.

\section{Fundamentals of Assistant Platform Ecosystems}

To set the stage, the basics for assistant platform ecosystems will be described. First, the understanding and properties of ecosystems are summarized and in a second step the current state of ecosystem intelligence and modelling. The chapter terminates with the present understanding of assistant platform ecosystems.

\subsection{Ecosystems}

Ecosystems are associations of organizations that are not hierarchically coordinated but interact in a modular way [5]. They are defined by the alignment structure needed by a multilateral set of actors to materialize a focal value proposition [2]. In ecosystem organizations, individuals and autonomous actors and systems jointly develop their capabilities and roles [9] with relationships combining aspects of competition and collaboration [10]. Since products and services are often complementary [10] ecosystem participants are linked by flows of data, services, and money [10]. The particular strength of ecosystems is that they provide a structure containing and coordinating complementarities without vertical integration [5]. Although ecosystems are often based on digital platforms, they are different concepts [10] [11] with the platform enabling the collaborations between multiple groups of actors in the ecosystem [12].

Although ecosystems can evolve in different directions, platform ecosystems have recently attracted much attention in research and practice [13]. Platform ecosystems are networks of innovation to produce complements that make a platform more valuable [14]. They embrace the platform sponsor and all firms that provide complementary offerings, which contribute to customer value [15]. Business ecosystems reflect the impact of the community of enterprises on a business. Innovation ecosystems enable firms to combine their offerings into a coherent customer-facing solution [16]. In addition, literature distinguishes other types of ecosystems (e.g. [2] and [5], and [17] in the information systems discipline).

The enterprises participating in an ecosystem are bound together because they cannot redeploy their collective investment elsewhere [5]. In ecosystems, the participating firms have a mutual interest in developing the ecosystem, although they compete and cooperate simultaneously [18]. The intricacies of value creation in ecosystems are analyzed in [15] and are often linked to service-dominant-logic (SDL). This perspective conceives ecosystems as self-contained and self-adaptive systems of loosely coupled resource-integrating actors connected by shared institutional logic and mutual value creation through service exchange [19]. The value cocreation mechanisms of business-to-business ecosystems are analyzed in [20] and with SDL in [21]. Due to their decentralized structure, ecosystems can leverage mechanisms such as social production [22] for innovation. The decentralized nature of ecosystems also explains their ability to continuously adapt and evolve, reacting to events from inside and outside [6] [21].

\subsection{Ecosystem Intelligence and Modelling}

The need to investigate ecosystem data in a structured way has been recognized in [23], and ecosystem intelligence has been suggested as a promising datadriven approach to understand ecosystems in [24]. Ecosystem intelligence is the structured analysis of ecosystem-related data to support decision-making, and only recently a call for more research on ecosystem intelligence was published [25]. Ecosystem-related data contains a broad range of entities, relationships, activities, and issues of interest [25]. Ecosystem intelligence and modeling are parts of empirical research on ecosystems reviewed in [26] and [27]. A framework of metrics to evaluate the performance of and the challenges to ecosystems is presented in [3]. It contains metrics to identify challenges for ecosystems during their lifecycle phases launch, scale, mature, and evolve. However, neither are details on data collection nor on processing provided, and only a few approaches use web mining to scrape unstructured information from enterprise websites (e.g. [28]).

For a long time, ecosystem analysis has suffered from a lack of structured data [4]. Instead, to collect data for ecosystem intelligence, a wide range of approaches are used, starting with analyzing enterprise websites to analyze the logo maps [29]. In [28], a web-mining approach is used to collect ecosystem data from enterprises. In [24], the authors combine a variety of independently created data sources on deals and alliance relationships, executive and funding relationships (EFRs), and public opinion and discourse (POD) to perform the 
analysis of ecosystems. Due to the diversity of the data sources and their independent evolutions, these approaches use data triangulation and visualization techniques [24] to connect information originating from different sources. Other intelligence approaches investigate API-based ecosystems [30].

A broad investigation of the different approaches was presented in [31] regarding the modeling of ecosystems. To analyze the ecosystem, it is represented as a network of nodes where edges may interconnect. Nodes and edges have attributes that describe their properties. Due to this inherent network structure, visualization plays an important role in supporting ecosystem intelligence. Visualization was proposed for understanding ecosystems [31] and a generalized approach was suggested in [32]. More specifically, it has been applied for visualizing interfirm relations in a mobile ecosystem [6] and [32] to reveal API ecosystems and enterprise strategy in [33].

\subsection{AI-based Assistant Platforms}

AI-based assistant platforms originate from voicebased systems that enable the interaction with an information system via the most natural form of communication, being the (spoken) human language [34]. Following the definition in [7], AI-based assistant platforms are digital platforms that inherit properties and dynamics from service as well as IoT platforms and yield new paths of value creation along the platform logic [1]. In the beginning, AI-based assistant platforms were constrained to few devices. However, increasingly, assistant platforms associate devices outside their original platform, such as TV sets and even microwave ovens [35]. Today, AI-based assistant platforms mediate access to an increasing number of services and devices via a voice-based interface [36]. The AI-based platform with the largest market share is Amazon Alexa closely followed by Google Assistant [37]. The Alexa platform enables interactions between users and several complementors with so-called skills. They link to external resources such as information and services, create a language-based interface [1] and are offered in the Alexa skills section of Amazon's website [38]. Skills differ from apps by being available on all devices after activation contrary to apps that have to be installed on every device. As argued in prior research [39], AI-based assistant platform ecosystems may be seen as a type of AIplatforms. For example, Alexa is embedded into the Amazon platform, thus pointing to the concept of "platforms of platforms" [40].

\section{Research Method}

To answer the three research questions, a five-step mixed-methods research methodology was developed. After searching for a suitable method for our research project, we investigated several earlier approaches on ecosystem intelligence (see section 2.2). These approaches collect aggregated information from multiple, independently evolving data sources, and therefore require substantial resources for manually merging semantically heterogeneous aggregated data. Although, only a human operator could ensure that the data collected from the different sources are merged into a consistent and coherent overall model, the methodology could take advantage of the highly digitalization of AIbased assistant platforms. Nearly all information on the Alexa ecosystem is explicitly or implicitly contained in the skill repository [41] or other digital sources, such as the analytics dashboard for Alexa developers [42].

Our hypothesis was that AI-based assistant platforms show the phenomenon of ontological reversal [43]: since they shape reality, research on them calls for special methods. We decided to use well-established social network approaches as presented in [44] and [45]. In addition, the present approach leverages the availability of a wealth of digital data on the ecosystems of AI-based assistant platforms that enables broadly applied practices of data collection and cleansing from machine learning [46] [47]. As shown in Fig. 1, the method comprised five phases with qualitative and quantitative techniques for raw data collection, construction of the conceptual data model, ecosystem elaboration, topological analysis, and value analysis. The research questions answered are shown on the right in Fig. 1.

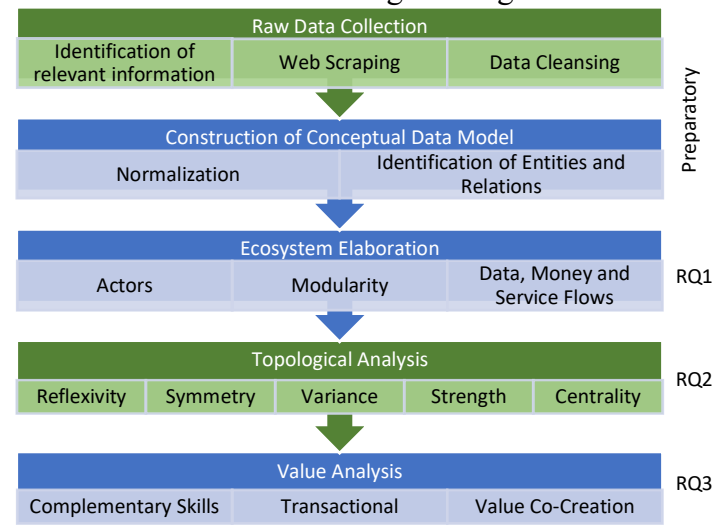

Fig. 1: Research Method (Phases in Green are Quantitative, Phases in Blue Qualitative Steps)

Information identified as relevant in the raw data collection phase was collected from the website through web scraping [48]. Since errors may occur by duplicate or missing entries during scraping, the collected data 
was processed in a second step. For example, we applied procedures based on the web page address to identify duplicate entries. Furthermore, we compared the entries with the typical intervals found in these data fields to detect outliers. Normalization rules of relational data model theory provided a solid foundation to guide the construction of a conceptual model of the ecosystem [49]. The ecosystem and its components were elaborated on the conceptual model, such as actors and relationships. The ecosystem was then represented graphically following established methods from social network research [50] [45]. Based on the graph-based representation of the ecosystem, we then identified properties of the topology of the ecosystem. After elaborating on the ecosystem and analyzing its topology, the results were used to investigate the value creation and co-creation in the ecosystem. Different methods, such as investigating complementarities were applied based on [5] as well as on the SDL for the value analysis [21].

\section{Ecosystem Intelligence for the Alexa Platform}

Following the research method in chapter 3, the adopted ecosystem intelligence approach includes the first four phases in Fig. 1. The first step comprises raw data capture, followed by the construction of the conceptual model in the second and the elaboration of the ecosystem in the third phase. The topology is part of the fourth phase.

\subsection{Raw Data Collection}

In raw data capture, data is collected from different data sources. The Amazon website provides the data in a special section on Alexa skills [41]. The data was collected from the German website because accessing the U.S. skill section created strange effects such as mixing the English skills with German text and the like. The similarity of the pages describing the Alexa skills with the regular Amazon product pages allowed to apply several techniques that were already available. We collected data during several web-scraping campaigns in April and May 2021 using a set of tools, which were selected based on comparisons of different web scraping tools such as [48]. Scrapy is a Python-based library for web scraping [51] and enables creating an automated bot that trawls web pages and scrapes them. For the collection of specific information, BeautifulSoup has been used [51]. By distributing the web-scraping campaigns to different users and different time slots, we could avoid the defensive actions of Amazon, such as blocking access to the Amazon website.

\subsection{Construction of Conceptual Model}

The structure of the scraped data is not geared towards the needs of a scientific evaluation but the technically efficient design of a website. The scraped data are flat files representing the website's content as csvfiles (Comma Separated Values) that contain structured and non-atomic, semi-structured, and even non-structured data. E.g., a string identifies the skills, called ASIN (denoting Amazon's Standard Identification Number). A list of ASINs defines the related skills squeezed into a string-typed field. Such non-atomic data had to be split up. Also, semi-structured data was contained. For example, there were standardized sentences in skill descriptions that defined the permissions required by the skill. Unstructured data was found in the free text forms, such as the field for customer reviews. The CSV files were transferred to a Python Jupyter notebook [52] containing Pandas [53] tables. We performed data cleansing operations such as detecting outliers as well as filling up missing values and, in addition, some relations between data had to be reconstructed.

\subsection{Ecosystem Elaboration}

The theoretical foundation of the ecosystem elaboration are the ecosystem definitions in [5] and [10]. After identifying the actors in the Alexa ecosystem, we analyzed the flows of data, services, and money in the ecosystem based on the definition in [10]. Following [5], a modular architecture [54] is an important but often overseen characteristic of ecosystems. Since modularity enables the coordination between organizations with significant autonomy, skills were identified as this module of interaction. Technically we transformed the table-oriented data created in the last step into a conceptual model using a graph-based representation. The conceptual model enabled us to apply proven methods from network research, such as in [44] and [45]. The graphbased representation was implemented with the help of Networkx [55], which offered the largest set of algorithms in comparison to other graph-oriented technologies such as graph-oriented databases. We carried out the analyses based on this graph-based representation using Jupyter notebooks.

Actors. We identified five types of actors in the Alexa ecosystem, depicted as rounded rectangles in Fig. 2 and connected by the service, data money flows. Skill publishers place skills onto the skill repository of the Alexa platform. A skill publisher may commission a skill-developer to create the skill. Device vendors provide access points and devices such as light switches. Access points also provide a gateway to many devices not directly attached to the internet, such as lightbulbs and switches. For example, skill providers overlap with 
device vendors in some skill categories in the smart home domain. The device vendors also provide skills to access, control, and monitor their devices.

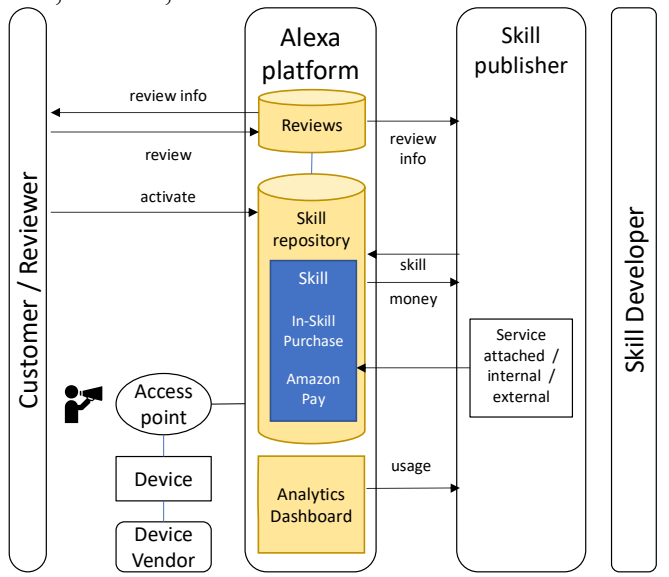

Fig. 2: Actors with Service, Data, and Money Flows

Customers select skills for activation and may be supported by review information. After activation, the skills are accessible to the customers for interaction. During skill usage, customers may enact in-skill purchases or arbitrary transactions based on Amazon Pay. This information is included in the textual skill description: the information about in-skill purchases and the access to user data is in the attribute "description". A reviewerId identifies customers who create reviews. The reviewers may also use a nickname.

Table 1: Actors and Related Entities in the Alexa Ecosystem

\begin{tabular}{|l|l|l|}
\hline Actor & $\#$ & Related entities \\
\hline Skill publishers & 3852 & Skills (10329) \\
\hline Reviewers & 61559 & Reviews (79211) \\
\hline Device vendors & 23 & Devices (102) \\
\hline
\end{tabular}

Reviews are associated with the reviewerId and identified by the reviewId. A review contains a rating of 1 to 5 stars and a text for the rating. A rating represents the average of the ratings, and the number of reviews is also given. Table 1 shows the number of entities in the Alexa ecosystem. Interestingly, there are some highly active publishers in the Alexa ecosystem and there is a high variance in the number of skills published. The most active publisher published 1595 skills, the second 402, and the third 196 skills.

Skills as Modules of Interaction. Modules are key elements in many ecosystems [5]. Skills are the modules of interaction in the Alexa ecosystem. Most interactions and flows reference a skill, as shown in Fig. 3. In the Alexa ecosystem, skills are identified by the ASIN, which as a unique identification number takes on the role of the primary key. Native skills are provided directly by Amazon. They are automatically activated without the action of the customer, such as weather forecasts and reminder functions.

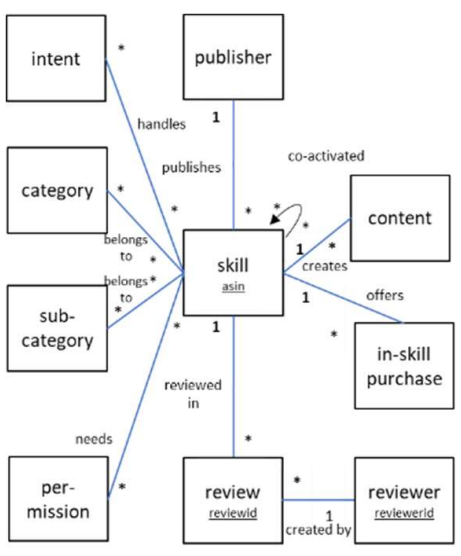

Fig. 3: Skills as Modules of Interaction

Non-native skills have to be activated before usage and belong to two classes [8]. First, custom skills enable the design of custom interaction models with arbitrary intents and utterances. Custom skills may be named arbitrarily and allow the definition of an interaction model containing a set of intents. They also provide control over the skill design and code. Second, there are predefined interaction models for several application domains containing intents and utterances: flash briefing, smart home, music, video, meetings, education, and custom. Flash briefing skills provide news feeds to the users that may customize the news updates. Smart home skills yield users control over light switches and other home equipment. Music skills provide the user with access to his music or music from streaming services. Video skills let users find and consume video content. To create meetings and book available meeting rooms, the meetings skill is defined. The education skill is for making the user know about upcoming coursework, school communications, and the like.

In the Alexa ecosystem, skills are associated with so-called categories. 23 top-level categories were found as depicted in Fig. 4 with some categories being further differentiated into sub-categories, which amount to 43 . During skill execution, Alexa maps the user's utterances to so-called intents, allowing different phrases to make requests to Alexa. An intent may contain so-called slots. Slots are named parameters for further processing. For example, an utterance requesting information on when trains are going from A to B may contain the departure time and destination slots. The Alexa platform maps the utterances to an intent and processes the request directly or via backend processing. After processing, a response to the user is created. The text is then translated to natural speech and transferred to the Alexa device. The response may also contain visual information or videos on appropriate devices such as the Amazon show device. By analyzing the attributes "description", "details", "inskill-purchases", "permissions" and "linked skills" we 
could gather additional information. The attribute "description" is used by many skill providers to describe the skill's functionality as intents and gives examples to access these intents. The attribute "linked skills" contains information about skills activated in common with the link (coactivation). The attribute "details" gives, among other things, information on the use of services. Skills may use embedded, internal or external services. Embedded services are part of the Alexa platform. Internal services are outside the platform but provided in the Amazon Web Services (AWS) cloud [56], which illustrates the concept of "platforms of platforms" [40]. External services are integrated via AWS but are external to AWS. The access to services is often indicated by the string "Contains data updated by the developer". The attribute "in skill-purchases" contains data on possible purchases from within the skill (see section 5.2). Some skills require access to user data such as location and the like. A skill may need authorizations, for example, to access to personal data. They are documented in the attribute "permissions". Skills are linked to other skills via the joint activation of coactivation. The skills can enable in-skill purchases and information on the skill also contains technical information such as links.

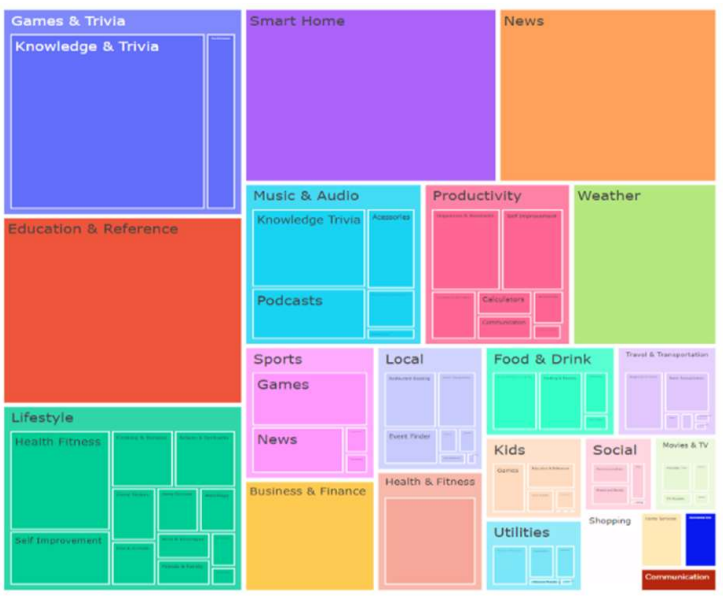

Fig. 4 : Skills per Category

Ecosystem Topology. The investigation of ecosystems based on their topology has already been suggested in [31]. We start by analyzing the coactivations of skills. There is a list of so-called linked skills that are most often activated together with the skill for each skill. We have examined the list of linked skills to see which categories the skills on the list belong to. We created a graph describing the strength of coactivations within and between the different categories. The five measures of the skill coactivations revealed:

- Reflexivity expresses the degree of coactivations within a category compared to the number of coactivations to other categories. There are only two cate- gories that have a very high intra-category coactivation. The categories "health \& fitness" and "music \& audio" had more coactivations with skills from their category than coactivations with any other category. At the same time, the category food \& drink had much fewer coactivations with skills of the same category than with other categories.

- The symmetry of coactivations denotes that the coactivations between skills are strong in both directions. For example, skills from the food \& drink category are often activated together with the category health \& fitness and vice versa. Interestingly, the number of coactivations in between two categories was not equal. There are also categories with highly asymmetric coactivations like skills of the category kids, which have more coactivations with skills from the music \& audio category than vice versa.

- Variance denotes the distribution of coactivations. On the one hand, some categories had many coactivations to only one or a few categories and the category food \& drink had many coactivations with health \& fitness, but only a few with other categories. On the other hand, the category education and reference had many coactivations with several other categories.

- Strength denotes the number of coactivations per skill in a category. Some categories, such as kids, featured many coactivations per skill, other categories such as smart home only a few. A hypothesis to explain this may be that the skills in the smart home category are often associated with specific hardware.

- Centrality expresses the contribution a node makes to the network's structure, i.e. how important the node is for the network [45]. The analysis of centrality is too complex to depict it here. However, Alexa showed an interesting anomaly since the centrality of the smart home category was very low. Further visual analysis showed that this category had only very few coactivations with other categories.

\section{Value Analysis of the Alexa Platform}

By analyzing actors, data, service, and money flows, and skills, three major value creation and capture models were found in the Alexa ecosystem: transactional business models, complementary services, and value-co-creation mechanisms.

\subsection{Complementary Skills}

Most skills in the Alexa ecosystem complement products and services, particularly in the smart home domain. These skills provide access to monitor or control physical devices integrated into the Alexa ecosystem or 
they provide information on digital services. Complementary skills may be differentiated according to the service lifecycle phase they support. For example, the phases information, negotiation, agreement, and service known from e-commerce may be appropriate here. In the insurance and transport systems sectors, the pre-purchase and post-purchase phases are the most supported. For example, the skill of the German railroads (bahn.de) provides information on train connections and the punctuality of running trains. Complementary skills increase resource liquefaction [21] by giving the customer information about a resource or the service. For example, they provide information about services, sensors, or actors, and may be seen as an accompanying supporting service or a meta-service.

\subsection{Transactional Business Models}

A small set of only 104 skills supported a transactional business model enabling transactions by selling goods or services. The transactional model was particularly widespread in the games and fun category where direct sales at fixed prices, usually in the form of a monthly subscription, are common. There were two transactional business models that may be distinguished. On the one hand, in-skill purchasing (ISP) [57] is used to sell digital goods and services. It supports three types of transactions: One-time purchases that provide access to premium content without expiration (entitlement), and subscriptions that provide access to premium content for some time can be prolonged or canceled (for an overview of the skills supporting entitlement and subscription ISP see Table 2. The payments received can be analyzed using the analytics dashboard. Finally, so-called consumable purchases yield access to depleted content when used and may be purchased again. All skills that use ISP need to be certified.

Table 2: Top-3-Categories of In-skill Purchases

\begin{tabular}{|l|l|l|l|}
\hline & Entitlement & Monthly & Yearly \\
\hline$\# 1$ & $\begin{array}{l}\text { Games \& Trivia } \\
(22)\end{array}$ & $\begin{array}{l}\text { Music \& Audio } \\
(80)\end{array}$ & $\begin{array}{l}\text { Games \& } \\
\text { Trivia (1) }\end{array}$ \\
\hline \#2 & Livestyle (6) & $\begin{array}{l}\text { Games \& } \\
\text { Trivia (22) }\end{array}$ & Utilities (1) \\
\hline \#3 & Food (6) & Health (9) & Weather (1) \\
\hline
\end{tabular}

On the other hand, Amazon Pay is used to sell realworld goods and services [57]. Two payment workflows are possible: charge now and charge later. The charge now workflow is intended for one-time purchases with an already known purchase amount. The charge later workflow best fits subscriptions, regular payments, and if the payment amount is not fixed yet. The latter is typical for upselling and pay-as-you-go usages. Up to now, only a few skills provide products or services using Amazon Pay. We found 11 examples such as selling insurance, travel, movie tickets, and fundraising.

\subsection{Value Co-Creation}

Our analysis of value co-creation in the Alexa ecosystem is based on the theoretical research of Nambisan and Lusch [21]. It builds on prior research that proposed a value co-creation-based conceptualization of assistant platforms [1]. Following this approach, AI-based assistant platforms are conceived to contribute to the efficiency and effectiveness of service exchange via resource liquefaction and increasing resource density [21]. Resource liquefaction is the detaching of information from resources that increase the transferability of resources [21]. Resource density describes whether the best available resources can be activated quickly to offer the desired service [21].

Analytics Dashboard. The skill publisher can access comprehensive metrics on the Alexa analytics dashboard [42]. It provides the number of skill activations and metrics on the customers' interaction, such as sessions, unique customers, utterances, and invoked intents. Also, the interaction path within the skill is provided, which describes the sequence of intents invoked. Two critical metrics are endpoint latency and endpoint response [42]: Endpoint latency measures the reaction time of backend processing. Endpoint response compares the number of successful interactions with the non-successful ones.

Review System. Customers also can share explicit feedback on their skills. A review system in the Skill Shop also enables them to rate the skill with 1 to 5 stars and to comment on the skill. As is generally the case, it is possible to rate the review in terms of its usefulness. The review information is available to the customers and the skill developer. The review system plays an important role in increasing resource density within the Alexa ecosystems. It provides supplementary information for selecting skills and thus assuring the proper selection of skills.

\section{Discussion and Contributions}

Ecosystems in general and the ecosystems around AI-based assistant platforms are becoming an important area for creating and enhancing digital business models. To properly design respective initiatives, it is necessary to understand the structure and status of the ecosystem. Therefore, this research adopted ecosystem intelligence on AI-based assistant platforms and formulated three research questions, which referred to the actors and relationships of AI-based assistant platforms, the topological properties of the ecosystem, and how value is created and co-created. A modified ecosystem intelligence approach was used to collect as much structured data as possible to address these research questions. Using data from Amazon's Alexa ecosystem, a conceptual model 
was elaborated for this ecosystem. Methodically, the digital platform itself was used as data source, which distinguishes this research from previous approaches that relied on indirect data sources and the extraction of human expertise. This allowed to address the ontological reversal [43] that identifies the information system as origin of transformation. Furthermore, it avoids the subjectivity of human observers introducing bias. There are connections to methodological research on using big data (e.g. [58] [59]) or web scraping [60] for information systems research. For a long time, information system research methods have used humans as a proxy for information system properties. Extensive surveys have been made asking human beings about the properties of information systems. This procedure is implicitly based on the assumption that the respondents are experts in the survey field and are also free of bias. But the certainty of classifying respondents as experts and their freedom from bias must be questioned since it presupposes the interviewer's expertise.

Several theoretical implications may be derived from this research may be seen as a contribution to the general development of methods for automated and timely analysis of rapidly evolving ecosystems. Compared to previous approaches, this allows richer and more comprehensive insights into the structure of digital ecosystems, for example, beyond determining the actors. In addition, the combination with the visualization of ecosystems seems promising. It adds to the body of existing techniques in the context ecosystem intelligence [32] inter-firm relationships [6] collaborative modeling [61] and visual analytics in API ecosystems [33]. It could lead to dedicated tools, such as an interactive tool that was already proposed for discovering, exploring, and analyzing business ecosystems [62]. In [3], a comprehensive set of key success metrics and red flags for identifying challenges for ecosystems during their launch, scale, mature, and evolve - lifecycle phases is identified. Since these contributions lack an analysis of ecosystem actors, topology, or value creation, the present research could prove valuable here. Our research has several practical implications. For example, important structures and interrelationships in the Alexa ecosystem are revealed and may be used by practitioners. Similar structures are certainly found in other ecosystems, such as that of Google Assistant. Also significant for practitioners is the low latency of our approach and its low resource requirements. Through automated data collection and analysis, we can quickly produce ecosystem analyses with relatively little effort, which can serve as a basis for strategy development and decision making.

\section{Conclusion, Limitations, and Outlook}

The major digital platform companies are known for applying AI in many ways, with prominent virtual assistants. Remarkably, both the application modules for AI and the assistants themselves have taken on the character of digital platforms. Whether from Amazon, Apple, or Google, an entire ecosystem has emerged in each major assistant platform. To date, little research has focused on analyzing these novel platforms. Data from the Alexa assistant platform provides the first insights into the structure of these platforms and how they create value. Starting with automatically-collected data, the present paper identified the players in the ecosystem and examined its topology. Skills were recognized as the unit of modularity in the Alexa ecosystem and play a central role. They generate data streams about reviews, ratings, and skill usage that customers and skill providers use. In addition to providing services, skills consume services such as Amazon Pay. This was mainly deducted from examining the topology of the Alexa ecosystem using the criteria of reflexivity, symmetry, variance, strength, and centrality of skill coactivations. Ecosystem intelligence and the value creation analysis of the Alexa ecosystem revealed many important insights. Skills create value as independent services, complementary services, and transactional models of value creation. In addition to providing services in isolation, skills often complement physical and services by providing information about them, configuring them, controlling them, and monitoring them. They support a transactional model of value creation and capture and a model of value collaboration. Analyzing skills via detailed monitoring of their use and collecting evaluations and ratings increases resource fluidity and density in the ecosystem. Future research will therefore deepen the study of these structures and extend them to other ecosystems. It might also address the limitations of our research. For example, including data from more countries would enrich the insights into the Alexa ecosystem. However, we are optimistic that the structures and relationships we identified are valid for other countries and languages by comparing structures and sampling. In addition, it would be interesting to learn about the evolution of the ecosystem over time. At present, we lacked access to important data such as the version history of the skills and lacked the space in this paper to present the results.

In summary, the ecosystem of AI-based assistant platforms is the foundation for new development paths of business, such as conversational [63] and voice-based commerce [64]. They differ from existing approaches by providing greater reach and a stronger linearity of customer interaction. Customers can interact as soon as they are in the vicinity of an access point, regardless of whether they have eye contact or not. The more linear 
structure requires a rethinking of the two-dimensional structure of traditional e-commerce, which, for example, can present alternatives simultaneously. Convenient access also makes conversational and voice-based commerce interesting for products that are familiar to customers and that have a low complexity of product description. For future research, it seems promising to intensify the investigation of skill reviews and ratings. However, parsing natural language and analyzing its sentiment requires a complete additional set of methods and tools, which will be covered in a separate paper. A further contribution of this research is to create the first sketch of a structured approach for analyzing the quickly evolving ecosystems following a network research approach and using automated data collection [50]. The adopted approach consumes significantly fewer resources and is much faster than existing approaches that require mandatory manual steps such as interviewing experts. It seems particularly suitable for rapidly evolving ecosystems and might pave the way to a new way to research in the field of information systems $[43][58][59][60]$.

\section{Acknowledgments}

We would like to thank our students from the class "IT-infrastructures" at Munich University of Applied Sciences to collect the raw data and provide them to us.

\section{References}

[1] Schmidt, R., R. Alt, and A. Zimmermann, "A Conceptual Model for Assistant Platforms", 54th Hawaii International Conference on System Sciences (HICSS), (2021), 4024-4033.

[2] Adner, R., "Ecosystem as Structure: An Actionable Construct for Strategy", Journal of Management 43(1), 2017, pp. 39-58.

[3] Pidun, U., M. Reeves, and E. Wesselink, "How Healty is Your Business Ecosystem ?", MIT Sloan Management Review 62(3), 2021, pp. 31-38

[4] Reeves, M., H. Lotan, J. Legrand, and M.G. Jacobides, "How Business Ecosystems Rise (and Often Fall)", MIT Sloan Management Review 60(4), 2019, pp. 1-8.

[5] Jacobides, M.G., C. Cennamo, and A. Gawer, "Towards a theory of ecosystems", Strategic Management Journal 39(8), 2018, pp. 2255-2276.

[6] Basole, R.C., "Visualization of Interfirm Relations in a Converging Mobile Ecosystem", Journal of Information Technology 24(2), 2009, pp. 144-159.

[7] de Reuver, M., C. Sørensen, and R.C. Basole, "The Digital Platform: A Research Agenda", Journal of Information Technology 33(2), 2018, pp. 124-135.

[8] “Alexa Developer Home", Amazon (Alexa). https://developer.amazon.com/alexa

[9] Vargo, S.L., and R.F. Lusch, "Institutions and Axioms: An Extension and Update of Service-Dominant Logic", Journal of the Academy of Marketing Science 44(1), 2016, pp. $5-23$.
[10] Fuller, J., M.G. Jacobides, and M. Reeves, "The Myths and Realities of Business Ecosystems", MIT Sloan Management Review 60(3), 2019, pp. 1-9.

[11] Asadullah, A., I. Faik, and A. Kankanhalli, "Digital Platforms: A Review and Future Directions", PACIS 2018 Proceedings, (2018), 15.

[12] Hagiu, A., "Strategic Decisions for Multisided Platforms", MIT Sloan Management Review 55(2), 2014, pp. 71-80.

[13] Alstyne, M.W.V., G.G. Parker, and S.P. Choudary, "Pipelines, Platforms, and the New Rules of Strategy", Harvard Business Review 94(4), 2016, pp. 54-62.

[14] Gawer, A., and M.A. Cusumano, Platform Leadership: How Intel, Microsoft, and Cisco Drive Industry Innovation, Harvard Business School Press, 2002.

[15] Ceccagnoli, Forman, Huang, and Wu, "Cocreation of Value in a Platform Ecosystem! The Case of Enterprise Software", MIS Quarterly 36(1), 2012, pp. 263-290.

[16] Adner, R., "Match Your Innovation Strategy to Your Innovation Ecosystem", Harvard Business Review 84(4), 2006, pp. 98-107.

[17] Guggenberger, T.M., F. Möller, T. Haarhaus, I. Gür, and B. Otto, "Ecosystem Types in Information Systems.", $E$ CIS, (2020).

[18] Moore, J.F., The Death of Competition: Leadership and Strategy in the Age of Business Ecosystems, Harper Business New York, 1996.

[19] Subramaniam, M., B. Iyer, and V. Venkatraman, "Competing in digital ecosystems", Business Horizons 62(1), 2019, pp. 83-94.

[20] Hein, A., J. Weking, M. Schreieck, M. Wiesche, M. Böhm, and H. Krcmar, "Value co-creation practices in business-to-business platform ecosystems", Electronic Markets 29(3), 2019, pp. 503-518.

[21] Lusch, R.F., and S. Nambisan, "Service Innovation: A Service-Dominant Logic Perspective", MIS Quarterly 39(1), 2015, pp. 155-175.

[22] Benkler, Y., The Wealth of Networks : How Social Production Transforms Markets and Freedom, Yale University Press, 2006.

[23] Basole, R.C., M. Hu, P. Patel, and J.T. Stasko, "Visual Analytics for Converging-Business-Ecosystem Intelligence", IEEE computer graphics and applications 32(1), 2011, pp. 92-96.

[24] Basole, R.C., M.G. Russell, J. Huhtamäki, N. Rubens, K. Still, and H. Park, "Understanding Business Ecosystem Dynamics: A Data-Driven Approach", ACM Transactions on Management Information Systems (TMIS) 6(2), 2015, pp. 1-32.

[25] Basole, R., "Understanding Ecosystem Data", Proceedings of the 53rd Hawaii International Conference on System Sciences, (2020), 5718-5727.

[26] Järvi, K., and S. Kortelainen, "Taking Stock of Empirical Research on Business Ecosystems: A Literature Review", International journal of business and systems research 11(3), 2017, pp. 215-228.

[27] Jussila, J., H. Kärkkäinen, S. Kortelainen, J. Huhtamäki, T. Aho, and T. Tebest, "New Era of Business Analytics: Making Sense of Business Ecosystems", Proceedings of the 18th International Academic MindTrek Conference, Association for Computing Machinery (2014), 266-268. 
[28] Kinne, J., and J. Axenbeck, "Web Mining for Innovation Ecosystem Mapping: A Framework and a Large-Scale Pilot Study", Scientometrics 125(3), 2020, pp. 2011-2041.

[29] Basole, R., "Mining Logomaps for Ecosystem Intelligence", Proceedings of the 54th Hawaii International Conference on System Sciences, (2021), 1081-1090.

[30] Basole, R., "The Ecosystem of Machine Learning Methods", (2021), 5871-5880

[31] Iyer, B.R., and R.C. Basole, "Visualization to Understand Ecosystems", Communications of the ACM 59(11), 2016, pp. 27-30.

[32] Basole, R.C., "Visual Business Ecosystem Intelligence", IEEE computer graphics and applications 34(5), 2014, pp. 26-34.

[33] Evans, P.C., and R.C. Basole, "Revealing the Api Ecosystem and Enterprise Strategy Via Visual Analytics", Communications of the ACM 59(2), 2016, pp. 26-28.

[34] Berdasco, A., G. López, I. Diaz, L. Quesada, and L.A. Guerrero, "User Experience Comparison of Intelligent Personal Assistants: Alexa, Google Assistant, Siri and Cortana", Proceedings 31(1), 2019, pp. 51.

[35] Chung, H., J. Park, and S. Lee, "Digital forensic approaches for Amazon Alexa ecosystem", Digital Investigation 22, 2017, pp. 15-25.

[36] López, G., L. Quesada, and L.A. Guerrero, “Alexa vs. Siri vs. Cortana vs. Google Assistant: A Comparison of Speech-Based Natural User Interfaces", In International Conference on Applied Human Factors and Ergonomics. Springer, Cham, 2017, 241-250.

[37] Vailshery, L., "Global Smart Speaker Market Share 2020", Statista, 2021. https://www.statista.com/statistics/792604/worldwide-smart-speaker-market-share/

[38] Anders, G., "Amazon's Alexa is a bet that in the future we will be talking to our computers", MIT Technology Review. https://www.technologyreview.com/s/608571/alexa-understand-me/

[39] Alt, R., "Electronic Markets on Digital Platforms and Ai", Electronic Markets 31(2), 2021, pp. 233-241.

[40] Nooren, P., N. van Gorp, N. van Eijk, and R.Ó. Fathaigh, "Should We Regulate Digital Platforms? A New Framework for Evaluating Policy Options", Policy \& Internet 10(3), 2018, pp. 264-301.

[41] "Alexa Skills", https://www.amazon.de/skills

[42] "Analytics for Alexa Development - Alexa Skills Kit Official Site", Amazon (Alexa). https://developer.amazon.com/en-US/alexa/alexa-skills-kit/get-deeper/analytics.html

[43] Baskerville, R.L., M.D. Myers, and Y. Yoo, "Digital First: The Ontological Reversal and New Challenges for Information Systems Research", MIS Quarterly 44(2), 2020, pp. 509-523.

[44] Scott, J., Social Network Analysis, SAGE Publications Ltd, London, 2012.

[45] Borgatti, S.P., M.G. Everett, and J.C. Johnson, Analyzing Social Networks, SAGE Publications Ltd, 2018.

[46] Raschka, S., and V. Mirjalili, Python Machine Learning: Machine Learning and Deep Learning with Python, scikit-learn, and TensorFlow 2, 3rd Edition, Packt Publishing, 2019.

[47] Provost, F., and T. Fawcett, Data Science for Business: What You Need to Know about Data Mining and Data- analytic Thinking, O'Reilly Media, Sebastopol, Calif., 2013.

[48] Gunawan, R., A. Rahmatulloh, I. Darmawan, and F. Firdaus, "Comparison of Web Scraping Techniques: Regular Expression, Html Dom and Xpath", ICoIESE 2018, Atlantis Press (2019), 283-287.

[49] Codd, E.F., "A Relational Model of Data for Large Shared Data Banks", In Pioneers and Their Contributions to Software Engineering. Springer, 2001, 61-98.

[50] Borgatti, S.P., and P.C. Foster, "The Network Paradigm in Organizational Research: A Review and Typology", Journal of Management 29(6), 2003, pp. 991-1013.

[51] Hajba, G.L., Website Scraping with Python Using BeautifulSoup and Scrapy, Springer, Cham, 2015.

[52] "Project Jupyter", https://www.jupyter.org

[53] "pandas - Python Data Analysis Library", https://pandas.pydata.org/

[54] Baldwin, C.Y., W.L.W.P. of B.A.C.Y. Baldwin, K.B. Clark, and P.K.B. Clark, Design Rules: The Power of Modularity, MIT Press, 2000.

[55] Hagberg, A., D. Schult, and P. Swart, "Exploring Network Structure, Dynamics, and Function Using Networkx", Proceedings of the 7th Python in Science Conference, Los Alamos National Lab.(LANL), Los Alamos, NM (United States) (2008), 11-16.

[56] "Amazon Web Services AWS - Server Hosting \& Cloud Services", https://aws.amazon.com/de/

[57] "Sell Products in an Alexa Skill", Amazon (Alexa). https://developer.amazon.com/en-US/alexa/techdoc-template.html

[58] Rizk, A., A. Ståhlbröst, and A. Elragal, "Data-driven innovation processes within federated networks", European Journal of Innovation Management ahead-ofprint(ahead-of-print), 2020.

[59] Kar, A.K., and Y.K. Dwivedi, "Theory Building with Big Data-Driven Research - Moving Away from the 'What' Towards the "Why", International Journal of Information Management 54, 2020, pp. 102205.

[60] Boegershausen, J., A. Borah, H. Datta, and A.T. Stephen, "Fields of Gold: Generating Relevant and Credible Insights Via Web Scraping and APIs", SSRN Electronic Journal, 2021.

[61] Faber, A., A. Hernandez-Mendez, S.-V. Rehm, and F. Matthes, "Collaborative Modelling and Visualization of Business Ecosystems", Australasian Journal of Information Systems 24, 2020.

[62] Basole, R., A. Srinivasan, H. Park, and S. Patel, "ecoxight: Discovery, Exploration, and Analysis of Business Ecosystems Using Interactive Visualization", $A C M$ Transactions on Management Information Systems 9, 2018, pp. 1-6.

[63] Rzepka, C., B. Berger, and T. Hess, "Why Another Customer Channel? Consumers' Perceived Benefits and Costs of Voice Commerce", Proceedings HICSS 53, (2020), 4079-4088.

[64] Piyush, N., T. Choudhury, and P. Kumar, "Conversational commerce a new era of e-business", 2016 International Conference System Modeling \& Advancement in Research Trends (SMART), IEEE (2016), 322-327. 